\title{
Does Compassion Fatigue Affect Nurse Educators in Practice?
}

\author{
Nida Mullins ${ }^{1, *}$, Laura McQueen $^{2}$ \\ ${ }^{1}$ School of Nursing, the University of North Carolina at Greensboro, United States \\ ${ }^{2}$ School of Nursing, the University of North Carolina at Chapel Hill, United States
}

Copyright $\mathrm{O} 2017$ by authors, all rights reserved. Authors agree that this article remains permanently open access under the terms of the Creative Commons Attribution License 4.0 International License

\begin{abstract}
Compassion fatigue is an unrecognized and understudied phenomenon that occurs in practicing nurses and most likely to affect nurse educators in practice. This unique form of burnout can impede nurse educators in caring for themselves and their students, patients, colleagues, schools and healthcare agencies. It is important to recognize the outcomes and implications of compassion fatigue and the growing need to mentor and care for both current and future nurse educators. Further research studies are needed on compassion fatigue in nurse educators to minimize the impact on educator health and professional nursing care.
\end{abstract}

Keywords Compassion Fatigue, Burnout, Nurse Educators, Stress Management

\section{Introduction}

Are you a nurse educator who has dreaded the thought of getting out of bed and going to clinical? Do you hate the idea of another exhausting 12-hour nursing shift with students? At times, have you at times lost all interest in working and caring for your patients and students? Have you actually thought about finding another job? If this sounds like your experience as an educator in nursing practice, you might be suffering from compassion fatigue.

The literature review identifies that nurses are suffering from fatigue, both, physically and mentally. However, there is little in the literature on nurse educators and compassion fatigue. Compassion fatigue is a unique form of burnout and occurs with the loss of nurturing capabilities that is essential to provide concerned nursing care. There is growing evidence of mounting distress and fatigue in the physical and psychological aspects of nurses including the lives of nurse educators. This article will focus on a literature review of compassion fatigue and explore the implications affecting nurse educators. This study will also consider strategies to recognize and manage compassion fatigue in nurse educators.

\section{Background}

Smart et al. (2013) [7] defines compassion fatigue as "the progressive and cumulative product of prolonged, continuous, and intensive contact with patients, and exposure to stress" (p.3). Healthcare workers provide care to their patients, and when they do this with compassion and empathy, they can enrich the lives of the patients and themselves, both professionally and personally. Compassion fatigue is the loss of the ability to nurture that is essential to providing compassionate care. The ability to feel for others erodes over time as normal stores of compassion are drained. When this happens, the quality of work and personal lives of healthcare workers are affected, and if left unchecked, can lead to lasting changes in healthcare workers' ability to provide compassionate care (Smart et. al., 2013) [7].

"Joinson (1992) a characterized a unique form of burnout as 'compassion fatigue.' She believed that because of it, nurses were experiencing forgetfulness, decreased attention span, exhaustion, physical illness, leading often to apathy and anger" (Ledoux, 2015, p. 2041) [4]. Compassion fatigue is often thought of as the caregiver's cost of caring. It results when caregivers are exposed to repeated interactions requiring high levels of empathic engagement with distressed clients: it can be a significant contributing factor in caregiver burnout (Sorenson, C., Bolick, B., Wright, K., and Hamilton, R, 2016, p. 457) [8].

Compassion fatigue manifests itself in a variety of physical, mental, emotional and work-related symptoms that affect patient care and relationships. Compassion fatigue occurs suddenly and is a direct result of another person's trauma. Exhaustion, avoidance of same or similar situations, hyperarousal, numbness and symptoms of post-traumatic stress disorder are noted in those suffering from compassion fatigue (Sorenson et al., 2016) [8]. Additionally, feelings of hopelessness, depersonalization, detachment and burnout have also been reported. 
Individual manifestations of compassion fatigue that are frequently reported include headaches, gastrointestinal issues, sleep disturbances, mood swings, irritability, depression, poor concentration and judgment, avoidance of particular situations and/or patients, diminished ability to feel empathy and loss of meaning in work. These manifestations often affect relationships with coworkers and patients (Sorenson et al., 2016) [8]. Ledoux identifies "the symptoms to becoming progressively worse to include questioning the meaning and purpose of their lives, isolating themselves from others, engaging in compulsive behaviors such as substance abuse, overspending, overeating, feeling apathetic, having difficulty concentrating and function in their roles" (Ledoux, 2015 p. 2043) [4]. Other possible indicators of compassion fatigue have been identified as chronic fatigue, muscle tension, cynicism, frustration, self-medication with alcohol, loss of interest in activities previously enjoyed, turnover, increased use of sick or vacation days, existential questioning and completely leaving the nursing profession (Boyle, 2015) [1].

Nurses working in the emergency room, critical care, progressive care unit, medical-surgical units and home health settings are at risk for the development of compassion fatigue. Healthcare professionals working in inpatient oncology settings reported higher rates of compassion fatigue when compared to their co-workers in an outpatient setting. Pediatric critical care nurses also reported high levels of compassion fatigue. According to Sorenson et al., (2016) [8]. "Almost $30 \%$ of pediatric nurses reported a high levels of burnout with more than $27 \%$ reporting a high level of compassion fatigue" (p.460). Nurses working with patients with Traumatic Brain Injuries (TBI) and burn units reported a higher level of compassion fatigue.

Paradoxically, healthcare personnel working in Hospice reported have smaller amount of compassion fatigue, as compared to chaplains who reported higher levels (Sorenson et. al., 2016) [8]. Nurses younger than 25 years old also reported higher incidences of compassion fatigue (Sorenson et. al., 2016). A study by Kelly, Runge and Spencer (2015) [3] also noted that the "Millennial" generation was more likely to experience higher levels of burnout. This study suggested that as nurses gained more experience, they were more likely to experience higher levels of compassion fatigue.

\section{Discussion}

Compassion fatigue has a strong relationship to burnout. According to Shirey (2006) [6] "Burnout is a prolonged response to chronic emotional and interpersonal stressors on the job that include 3 dimensions of exhaustion, cynicism (depersonalization), and inefficacy" (p.95). Individuals experiencing physical signs of burnout exhibit lack of energy, being tired during the day and the inability to sleep at night. Depression, helplessness, and hopelessness are emotions manifestations of burnout. Mental exhaustion is displayed in form of a negative attitude toward work and life, impatient with others, a displays a cynical reaction to developing problems (Shirey, 2006) [6].

The literature identifies that the lack of nurse managerial support in emergency departments and critical care has contributed to burnout and compassion fatigue (Sorenson et.al., 2016) [8]. Powerlessness, physical distress, values conflicts, medication errors, violence, and death were also factors for critical care nurses (Sorenson et al., 2016) [8]. Compassion fatigue was positively related to burnout and inpatient nurses' intention to leave the profession (Sorenson et al., 2016) [8]. These findings are worrisome in light of the continued nursing shortage, an aging population, and population growth. Another associated consequence of compassion fatigue to the healthcare organization is low patient satisfaction scores (Kelley, Runge \& Spencer 2015) [3].

\section{Implications}

How do nurses guard against the developing of compassion fatigue? Self-care practices are the most significant preventative measures identified by healthcare professionals to protect nurses from the development of compassion fatigue. Other interventions to prevent the development of compassion fatigue include education on coping mechanisms and teamwork. Because poor administrative leadership is found to be a contributing factor to compassion fatigue and burnout, managers will need to exercise a professional environment that promotes teamwork among nurses and positive working relationships (Sorenson et. al., 2016) [8].

Nursing faculty experience stress related to the high demands of teaching, research, and service. Other stressors identified by nursing faculty include heavy workloads, pressure to continue to maintain clinical competence and attempting to satisfy multiple agencies. To combat burnout among nursing faculty strategies such as mentoring programs, renewal techniques that promote a hopeful future and building a sense of community at the college or university and organizational engagement efforts such as appropriate recognition and rewards for a job well done. Faculty also need a supportive work environment that allows and encourages choice and control (Shirey, 2006) [6].

According to Brown (2016) [2] employers should implement the following to combat the development of compassion fatigue; educate managers on the early signs of compassion fatigue (particularly behavioral changes) and how to identify them; implement training programs to spot stress and anxiety among healthcare workers; encourage a culture of understanding for those employees suffering from compassion fatigue; offer employee assistance programs to allow for employees to verbalize their feelings and include wellbeing initiatives to help them cope with stressful or traumatic situations in which they have been involved or 
witnessed. According to May (2016) [5], other strategies to manage compassion fatigue include onsite counseling, the use of art therapy, and scheduling frequent breaks for healthcare workers.

Boyle (2015) [1] identified support groups, debriefing sessions, chair massage, aromatherapy, journaling, meditation and prayer as additional methods to effectively manage compassion fatigue in the workplace. Other strategies identified by Boyle (2015) [1] include taking care of yourself by engaging in good nutrition, exercising regularly, reducing stress, participating in social events, taking up a hobby, and nurturing spiritual needs.

\section{Summary}

Compassion fatigue has been studied in healthcare settings with direct care healthcare providers, but these studies did not include nurse educators. Can it be implicit that nurse educators can also suffer from what is recognized as compassion fatigue? Nurse faculty often spend many hours each week with students taking care of multiple patients and many work as nurses in these same healthcare setting. In addition to caring about patient care and their health concerns, educators also care for their student's success and struggles while in nursing school. Faculty work closely with students are affected when a student fails an exam, makes a medication error or charts incorrectly, or when a student uses non therapeutic communication to a patient and the patient becomes upset. Nurse educators are present when a registered nurse becomes angry at the student for not performing as an experienced nurse. These educators are also present when students have personal problems that are interfering with their nursing education or when a student receives word of acceptance and declination letters to a desired nursing program.

As nursing educators, we "care" for our students, we have compassion for them, or we would most likely not be teaching. The topic of caring in nursing has been one of extensive study and writing. It is a core value that supports nursing practice (Ledoux, 2015). As Watson (2006) states, "it is the caring relationship that often calls individuals into nursing in the first place" (Ledoux, 2015, p. 2046). The outcome of caring too much or giving to others to the detriment of the provider of care is an important topic of discourse for nurse educators. As nursing students prepare for the role of the nurse to provide care with compassion and empathy they must be able to differentiate when their lives are enriched through this care and when they are at risk of developing compassion fatigue. Likewise, nurse educators must also be able to differentiate when they are at risk of failing in their nurturing capability that is essential to providing compassionate care to their students, patients, co-workers, institutions, and themselves.

\section{Conclusions}

Compassion fatigue has had little recognition and even less study in the forum and practice of those who teach nursing students. It is probable that in the coming years as experienced nurse educators retire from the profession, there will be a growing need to assist future generations of nurse educators' with both the physical and mental fatigue inherent in nursing education. It is important to recognize the ways that compassion fatigue has an impact on the retention of nurse educators. Further recognition and study on compassion fatigue is needed to safeguard educators and minimize the impact to their health and to professional nursing care.

\section{Acknowledgements}

Peggy Markum, MSLS, Independent Editorial Consultant, The University of North Carolina at Greensboro, Greensboro, NC 27402

Ghaith Melhen, Research Assistant, The University of North Carolina at Greensboro, Greensboro, NC 27402

\section{REFERENCES}

[1] Boyle, D. A. (2015). Compassion fatigue: The cost of caring. Nursing2015, 45(7), 48-51.doi:10.1097/01.nurse.0000461857.48809.a1

[2] Brown, S. \& Nowlan, K. (2016). Cutting the risk of compassion fatigue. Occupational Health \& Wellbeing, 68(8), 24-25. Retrieved from

http://search.proquest.com/docview/1819114549?pq-origsite $=$ gscholar

[3] Kelly, L., Runge, J., \& Spencer, C. (2015). Predictors of compassion fatigue and compassion satisfaction in acute care nurses. Journal of Nursing Scholarship, 47(6), 522-528. doi: 10.1111/jnu.12162

[4] Ledoux, K. (2015). Understanding compassion fatigue: understanding compassion. Journal of Advanced Nursing, 71(9), 2041-2050. doi: 10.1111/jan.12686

[5] May, S. (2016). Combating compassion fatigue. Florida Nurse, 64 (3), 15. Retrieved from http://www.floridanurse.org

[6] Shirey, M. R. (2006). Stress and burnout in nursing faculty. Nurse Educator, 31(3), 95-97.

[7] Smart, D., English, A., James, J., Wilson, M. Daratha, K. B., Childers, B., \& Magera, C. (2014). Compassion fatigue and satisfaction: A cross - sectional survey among US healthcare workers. Nursing \& Health Sciences, 16(1), 3-10. doi: 10.1111/nhs. 12068

[8] Sorenson, C., Bolick, B., Wright, K., \& Hamilton, R. (2016). Understanding compassion fatigue in healthcare providers: A review of current literature. Journal of Nursing Scholarship, 48(5), 456-465. doi: 10.1111/jnu.12229. 